VOL. $15(1976), 431-438$.

\title{
A Banach space of functions of generalized variation
}

\section{A.M. Russell}

In this note we show that $B V_{k}[a, b]$, the linear space of

functions of bounded $k$ th variation on $[a, b]$, is a Banach

space under the norm $\|\cdot\|_{k}$, where

$$
\|f\|_{k}=\sum_{s=0}^{k-1}\left|f^{(s)}(a)\right|+V_{k}(f ; a, b) .
$$

\section{Introduction}

It is well known that $B V[a, b]$, the class of functions of bounded variation on $[a, b]$ is a Banach space under the norm $\|\cdot\|_{I}$, where

$$
\|f\|_{1}=|f(a)|+V_{1}(f ; a, b) .
$$

We generalize this result by showing that when $k$ is an integer greater than one, $B V_{k}[a, b]$ is a Banach space under the norm $\|\cdot\|_{k}$, where

$$
\|f\|_{k}=\sum_{s=0}^{k-1}\left|f^{(s)}(a)\right|+v_{k}(f ; a, b),
$$

and where, for convenience of notation, $f^{(s)}(a)$ means $f_{+}^{(s)}(a)$, and $f_{+}^{(s)}=\left(f_{+}^{(s-1)}\right)_{+}^{1}$. The definitions of $B V_{k}[a, b]$ and $V_{k}(f) \equiv V_{k}(f ; a, b)$

Received 21 July 1976. In a recent private communication the author has learnt that Dr Frank Huggins, University of Texas at Arlington, has shown, in particular, that $B V_{2}[a, b]$ is a Banach space under $\|\cdot\|_{2}$. He thanks him for this communication. 
can be found in Russell [1].

We also take the opportunity to improve some results of Russell [1]. In particular we present a sharper version of Theorem 4, and take this opportunity to point out that "a set of measure zero" can be replaced by "a countable set" in Theorem 12.

\section{Preliminaries}

We readily observe that $\|\cdot\|_{k}$ satisfies all properties of a norm except possibly that $\|f\|_{k}=0$ implies $f=0$. Accordingly, if $\|f\|_{k}=0$, then $V_{k}(f ; a, b)=0$, and this implies that

$$
Q_{k}\left(f ; x_{i}, \ldots, x_{i+k}\right)=0
$$

for any $(k+1)$ points $x_{i}, \ldots, x_{i+k}$ in $[a, b]$. Using a well known property of divided differences, we conclude that $f$ must be a polynomial of degree $(k-1)$ at most. That $f=0$ now follows readily.

We now improve our characterization of $B V_{k}[a, b]$. In Russell [1] it was shown that

$$
\begin{aligned}
& B V_{k}[a, b]=\left\{f: f=f_{1}-f_{2} \text {, where } f_{1} \text { and } f_{2}\right. \text { are } \\
& 0-, 1-\ldots, k \text {-convex functions having right and left }(k-1) \text { th } \\
& \text { Riemann * derivatives at } a \text { and } b \text { respectively }\}
\end{aligned}
$$

If $k=2$ it follows immediately that the $(k-1)$ th Riemann * derivatives at $a$ and $b$ can be replaced by the usual right and left hand derivatives respectively. Assume now that $k \geq 3$, and that $f \in B V_{k}[a, b]$. Then according to Theorem 12 of Russell [1], $f^{(k-2)}$ is continuous on $[a, b]$ and belongs to $B V_{2}[a, b]$. Thus $f_{+}^{(k-1)}(a)$ and $f_{-}^{(k-1)}(b)$ must exist. We summarize the previous discussion in the following

THEOREM 1. If $k$ is an integer greater than or equal to one, then $B V_{k}[a, b]=\left\{f: f=f_{1}-f_{2}\right.$, where $f_{1}$ and $f_{2}$ are $0-, 1-, \ldots, k$-convex functions having right and left $(k-1)$ th derivatives at $a$ and $b$ respectively\}. 
Our next result is an improved version of Theorem 4 of Russell [1].

THEOREM 2. If $f \in B V_{k+I}[a, b]$, and $k \geq 0$, then $Q_{k}\left(f ; y_{0}, y_{1}, \ldots, y_{k}\right)$ is bounded when $a \leq y_{i} \leq b, i=0,1, \ldots, k$. More precisely,

(1) $\left|Q_{k}\left(f ; y_{0}, y_{1}, \ldots, y_{k}\right)-Q_{k}\left(f ; a_{0}, a_{1}, \ldots, a_{k}\right)\right| \leq v_{k+1}(f ; a, b)$, where $a_{0}, a_{1}, \ldots, a_{k}$ is a fixed $\pi$ subdivision of $[a, b]$.

proof. Let $a_{0}, a_{1}, \ldots, a_{k}$ be a fixed $\pi$ subdivision of $[a, b]$, and let $y_{0}, y_{1}, \ldots, y_{k}$ be another $\pi$ subdivision of $[a, b]$ such that $a_{0}<\ldots<a_{k-1}<y_{0}<a_{k}<y_{1}<\ldots<y_{k}$. Re-label the points $a_{0}, \ldots, a_{k}, y_{0}, \ldots, y_{k}$ as $x_{0}, x_{1}, \ldots, x_{2 k+1}$, where

$$
\begin{aligned}
x_{i} & =a_{i}, i=0,1, \ldots, k-1, \\
x_{k} & =y_{0}, \\
x_{k+1} & =a_{k}, \\
x_{i} & =y_{i-k-1}, i=k+2, \ldots, 2 k+1 .
\end{aligned}
$$

Using Theorem 1 of Russell [1], we obtain

$$
\begin{aligned}
& Q_{k}\left(f ; y_{0}, y_{1}, \ldots, y_{k}\right)-Q_{k}\left(f ; a_{0}, a_{1}, \ldots, a_{k}\right) \\
& =\alpha_{1} Q_{k}\left(f ; y_{0}, a_{k}, y_{1}, \ldots, y_{k-1}\right)+\beta_{1} Q_{k}\left(f ; a_{k}, y_{1}, \ldots, y_{k}\right) \\
& \quad-\alpha_{2} Q_{k}\left(f ; a_{0}, a_{1}, \ldots, a_{k-1}, y_{0}\right)-\beta_{2} Q_{k}\left(f ; a_{1}, \ldots, a_{k-1}, y_{0}, a_{k}\right) \\
& \quad \text { where } \alpha_{1}+\beta_{1}=1=\alpha_{2}+\beta_{2} \\
& =\alpha_{1} Q_{k}\left(f ; x_{k}, x_{k+1}, \ldots, x_{2 k}\right)+\beta_{1} Q_{k}\left(f ; x_{k+1}, \ldots, x_{2 k+1}\right) \\
& \quad-\alpha_{2} Q_{k}\left(f ; x_{0}, x_{1}, \ldots, x_{k}\right)-\beta_{2} Q_{k}\left(f ; x_{1}, \ldots, x_{k+1}\right) \\
& =\left[Q_{k}\left(f ; x_{k}, \ldots, x_{2 k}\right)-Q_{k}\left(f ; x_{0}, \ldots, x_{k}\right)\right] \\
& \quad+\beta_{1}\left[Q_{k}\left(f ; x_{k+1}, \ldots, x_{2 k+1}\right)-Q_{k}\left(f ; x_{k}, \ldots, x_{2 k}\right)\right] \\
& \quad+\beta_{2}\left[Q_{k}\left(f ; x_{0}, \ldots, x_{k}\right)-Q_{k}\left(f ; x_{1}, \ldots, x_{k+1}\right)\right]
\end{aligned}
$$




$$
\begin{aligned}
=\sum_{i=1}^{k}\left[Q _ { k } \left(f ; x_{i}, \ldots,\right.\right. & \left.\left.x_{i+k}\right)-Q_{k}\left(f ; x_{i-1}, \ldots, x_{i+k-1}\right)\right] \\
& +\beta_{1}\left[Q_{k}\left(f ; x_{k+1}, \ldots, x_{2 k+1}\right)-Q_{k}\left(f ; x_{k}, \ldots, x_{2 k}\right)\right] \\
& +\beta_{2}\left[Q_{k}\left(f ; x_{0}, \ldots, x_{k}\right)-Q_{k}\left(f ; x_{1}, \ldots, x_{k+1}\right)\right] \\
=\alpha_{2}\left[Q _ { k } \left(f ; x_{1}, \ldots,\right.\right. & \left.\left.x_{k+1}\right)-Q_{k}\left(f ; x_{0}, \ldots, x_{k}\right)\right] \\
& +\sum_{i=2}^{k}\left[Q_{k}\left(f ; x_{i}, \ldots, x_{i+k}\right)-Q_{k}\left(f ; x_{i-1}, \ldots, x_{i+k-1}\right)\right] \\
& +\beta_{1}\left[Q_{k}\left(f ; x_{k+1}, \ldots, x_{2 k+1}\right)-Q_{k}\left(f ; x_{k}, \ldots, x_{2 k}\right)\right] .
\end{aligned}
$$

Taking absolute values now, and noting that $0 \leq \alpha_{2} \leq 1,0 \leq \beta_{1} \leq 1$, gives the required inequality.

An argument similar to that above establishes ( 1 ) in cases corresponding to other relative distributions of the sets of points $y_{0}, \ldots, y_{k}$ and $a_{0}, \ldots, a_{k}$.

COROLLARY. If $f \in B V_{k+1}[a, b]$, and $k \geq 0$, then

(2) $\sup _{\pi}\left|Q_{k}\left(f ; x_{0}, \ldots, x_{k}\right)\right|-\inf _{\pi}\left|Q_{k}\left(f ; x_{0}, \ldots, x_{k}\right)\right| \leq V_{k+1}(f ; a, b)$.

REMARK. The inequality (2) is best possible as illustrated by the case $k=1, a=0, b=1$, and

$$
f(x)= \begin{cases}0, & 0 \leq x \leq \frac{7}{2}, \\ x-\frac{1}{2}, & \frac{1}{2} \leq x \leq 1 .\end{cases}
$$

THEOREM 3. If $f \in B V_{k+1}[a, b]$, and $k \geq 0$, then $f \in B V_{k}[a, b]$, and

$$
V_{k}(f ; a, b) \leq k(b-a)\left[V_{k+1}(f ; a, b)+\inf _{\pi}\left|Q_{k}\left(f ; x_{0}, \ldots, x_{k}\right)\right|\right] .
$$

Proof. The first part of the theorem follows from Theorem 10 of Russell [1].

For the second part, it follows from Theorem 2, Corollary, that for any $\pi$ subdivision $x_{i}, \ldots, x_{i+k}$ of $[a, b]$, 


$$
\begin{aligned}
\left(x_{i+k}-x_{i}\right) \mid Q_{k}\left(f ; x_{i},\right. & \left.\ldots, x_{i+k}\right) \mid \\
& \leq\left(x_{i+k}-x_{i}\right)\left[v_{k+1}(f ; a, b)+\inf _{\pi}\left|Q_{k}\left(f ; x_{0}, \ldots, x_{k}\right)\right|\right] .
\end{aligned}
$$

Summing from $i=0$ to $i=n-k$, and taking the supremum gives (3).

REMARK. The constant in (3) is best possible as illustrated by the case $k=1, a=0, b=1, f(x) \equiv x$.

\section{Main results}

THEOREM 4. If $\left\{g_{n}\right\}$ is a sequence of functions in $B V_{k+1}[a, b]$, $k \geq 0$, such that $\left\|g_{n}\right\|_{k+1} \rightarrow 0$ as $n \rightarrow \infty$, then $\left\|g_{n}\right\|_{k} \rightarrow 0$ as $n \rightarrow \infty$.

Proof. It follows immediately from Theorem 10 of Russell [1] that $g_{n} \in B V_{k}[a, b]$ for all $n$. Since $\left\|g_{n}\right\|_{k+1} \rightarrow 0$ as $n \rightarrow \infty$, given $\varepsilon>0$, there exists $N(\varepsilon)$ such that

$$
\left\|g_{n}\right\|_{k+1}<\varepsilon
$$

whenever $n>N(\varepsilon)$. Hence, whenever $n>N(\varepsilon)$,

$$
\sum_{s=0}^{k}\left|g_{n}^{(s)}(a)\right|<\varepsilon,
$$

and

$$
V_{k+1}\left(g_{n} ; a, b\right)<\varepsilon
$$

Since $g_{n} \in B V_{k+1}[a, b],\left|g_{n}^{(k)}(a)\right|$ exists and is less than $\varepsilon$ whenever $n>N(\varepsilon)$, by (4). Hence, whenever $n>N(\varepsilon)$,

$$
\inf _{\pi}\left|Q_{k}\left(g_{n} ; x_{0}, \ldots, x_{k}\right)\right|<2 \varepsilon k ! \text {. }
$$

It now follows from Theorem 3 that $V_{k}\left(g_{n} ; a, b\right) \rightarrow 0$, and hence that $\left\|g_{n}\right\|_{k} \rightarrow 0$ as $n \rightarrow \infty$.

We now consider a Cauchy sequence $\left\{f_{n}\right\}$ in $B V_{k}[a, b]$. Consequently, for each $\varepsilon>0$, there exists $N(\varepsilon)$ such that 


$$
\sum_{s=0}^{k-1}\left|f_{m}^{(s)}(a)-f_{n}^{(s)}(a)\right|+v_{k}\left(f_{m}-f_{n} ; a, b\right)<\varepsilon,
$$

whenever $m, n$ exceed $N(\varepsilon)$.

If $\left\{f_{n}\right\}$ is a Cauchy sequence in $B V_{k}[a, b]$, it follows from Theorem 4 that $\left\{f_{n}(x)\right\}$ is a Cauchy sequence for each $x \in[a, b]$. Accordingly, we define

$$
f(x)=\lim _{n \rightarrow \infty} f_{n}(x), x \in[a, b] .
$$

THEOREM 5. If $f_{n} \in B V_{k}[a, b]$ for alz $n$, and $f(x)=\lim _{n \rightarrow \infty} f_{n}(x)$, $x \in[a, b]$, then $f \in B V_{k}[a, b]$.

Proof. Let $S_{\pi}(f)$ denote an approximating sum for $V_{k}(f ; a, b)$. Let $\left\{f_{n}\right\}$ be a Cauchy sequence in $B V_{k}[a, b]$, so that for each $\varepsilon>0$, there exists $N(\varepsilon)$ such that $\left\|f_{m}-f_{n}\right\|_{k}<\varepsilon$ whenever $m$ and $n$ exceed $N(\varepsilon)$. Therefore, whenever $m, n$ exceed $N(\varepsilon)$, $S_{\pi}\left(f_{m}-f_{n}\right)$

$$
=\sum_{i=0}^{n-k}\left|Q_{k-1}\left(f_{m}-f_{n} ; x_{i}, \ldots, x_{i+k-1}\right)-Q_{k-1}\left(f_{m}-f_{n} ; x_{i+1}, \ldots, x_{i+k}\right)\right|<\varepsilon
$$

for all $\pi$ subdivisions of $[a, b]$. Letting $m \rightarrow \infty$ in the last inequality gives

$$
S_{\pi}\left(f-f_{n}\right) \leq \varepsilon
$$

for all. $\pi$ subdivisions of $[a, b]$, and whenever $n>N(\varepsilon)$. Let $n_{0}$ be a fixed integer exceeding $N(\varepsilon)$, and let $\sup _{\pi} S_{\pi}\left(f_{n_{0}}\right)=K_{n_{0}}$. Then

$$
S_{\pi}(f) \leq S_{\pi}\left(f-f_{n_{0}}\right)+S_{\pi}\left(f_{n_{0}}\right) \leq \varepsilon+K_{n_{0}}
$$

for all $\pi$ subdivisions of $[a, b]$. Hence $f \in B V_{k}[a, b]$, as required. It now remains to show that $\left\|f_{n}-f\right\|_{k} \rightarrow 0$ as $n \rightarrow \infty$; that is, that 


$$
\sum_{s=0}^{k-1}\left|f_{n}^{(s)}(a)-f^{(s)}(a)\right|+v_{k}\left(f_{n}-f\right) \rightarrow 0 \text { as } n \rightarrow \infty \text {. }
$$

It is clear that $V_{k}\left(f_{n}-f\right)$ and $f_{n}(a)$ both converge to 0 as $n \rightarrow \infty$, so we now show that $f_{n}^{(s)}(a)-f^{(s)}(a) \rightarrow 0$ as $n \rightarrow \infty$ when $s=1,2, \ldots, k-1$.

We first observe that $f^{(s)}(a)$ exists when $s=1,2, \ldots, k-1$, because $f \in B V_{k}[a, b]$. Let $s=k-1$. Then it follows from Theorem 12 of Russell [1] that $f^{(k-1)}(x)$ exists on $[a, b]$, except possibly on a countable set. For each $n$, let $A_{n}=\left\{x: f_{n}^{(k-1)}(x)\right.$ exists $\}$, so that $[a, b] \backslash A_{n}$ is countable. Let $x>a$, and $x \in A=\prod_{1}^{\infty} A_{n}$. Since $v_{k}\left(f_{m}-f_{n}\right)<\varepsilon$ whenever $m, n$ exceed $N(\varepsilon)$, $Q_{k-1}\left(f_{m}-f_{n} ; x, x+h, \ldots, x+(k-1) h\right)$

$$
-Q_{k-1}\left(f_{m}-f_{n} ; a, a+h, \ldots, a+(k-1) h\right) \mid<\varepsilon,
$$

for all $\pi$ subdivisions of $[a, b]$ such that $a+(k-1) h<x$. Letting $h \rightarrow 0$ gives

$$
\left|\left[f_{m}^{(k-1)}(x)-f_{n}^{(k-1)}(x)\right]-\left[f_{m}^{(k-1)}(a)-f_{n}^{(k-1)}(a)\right]\right| \leq(k-1) ! \varepsilon,
$$

whenever $x \in A$ and $m, n$ exceed $N(\varepsilon)$. Therefore, using (6), it follows that

$$
\begin{aligned}
\left|f_{m}^{(k-1)}(x)-f_{n}^{(k-1)}(x)\right| & \leq(k-1) ! \varepsilon+\left|f_{m}^{(k-1)}(a)-f_{n}^{(k-1)}(a)\right| \\
& <(k-1) ! \varepsilon+\varepsilon,
\end{aligned}
$$

whenever $x \in A$ and $m, n$ exceed $N(\varepsilon)$. Thus $\left\{f_{n}^{(k-1)}(x)\right\}$ converges uniformly to $\phi(x)$, say, on $A$.

Since $f_{n}^{(k-2)}$ is absolutely continuous on $[a, b]$, it follows that

$$
f_{n}^{(k-2)}(x)-f^{(k-2)}(a)=\int_{a}^{x} f_{n}^{(k-1)}(t) d t
$$


and hence that

$$
f^{(k-2)}(x)-f^{(k-2)}(a)=\int_{a}^{x} \phi(t) d t .
$$

Consequently $f^{(k-1)}(x)$ exists, almost everywhere, and equals $\phi(x)$. In particular,

$$
f^{(k-1)}(a)=\lim _{n \rightarrow \infty} f_{n}^{(k-1)}(a)
$$

Similarly,

$$
f^{(s)}(a)=\lim _{n \rightarrow \infty} f_{n}^{(s)}(a) \text { when } s=1,2, \ldots, k-2
$$

Returning to (7) we see that $\left\|f_{n}-f\right\|_{k} \rightarrow 0$ as $n \rightarrow \infty$, and so we conclude that $B V_{k}[a, b]$ is a Banach space under $\|\cdot\|_{k}$.

\section{Reference}

[1] A.M. Russell, "Functions of bounded kth variation", Proc. London Math. Soc. (3) 26 (1973), 547-563.

Department of Mathematics,

University of Melbourne,

Parkville,

victoria. 